\title{
Proper manual handling techniques to prevent low back pain, a Cochrane Systematic Review
}

\author{
Jos H. Verbeek ${ }^{\mathrm{a}}$, Kari-Pekka Martimo ${ }^{\mathrm{a}}$, P. Paul F.M. Kuijer ${ }^{*}$, Jaro Karppinen ${ }^{\text {a }}$, Eira Viikari-Juntura ${ }^{\mathrm{a}}$ \\ and Esa-Pekka Takala ${ }^{\mathrm{a}}$ \\ ${ }^{a}$ Finnish Institute of Occupational Health, PO Box 31070701 Kuopio, Finland \\ ${ }^{b}$ Coronel Institute of Occupational Health, Academic Medical Center, University of Amsterdam, PO Box 22700 , \\ 1100 DE Amsterdam, the Netherlands
}

\begin{abstract}
Training and provision of assistive devices are considered major interventions to prevent and treat low back pain (LBP) among workers exposed to manual material handling (MMH). To establish the effectiveness of training and provision of assistive devices in preventing and treating LBP an update of a Cochrane literature review was performed to November 2010. Randomised controlled trials (RCTs) and cohort studies with a concurrent control group (CCTs) were included. Nine RCTs (20.101 employees) and nine CCTs (1280 employees) were included for prevention: six more than in the previous version. No study on treatment was found. None of the included RCTs and CCTs provided evidence that training and provision of assistive devices prevented LBP when compared to no intervention or another intervention.
\end{abstract}

Keywords: prevention, musculoskeletal, lifting, carrying, training.

\section{Introduction}

Manual material handling (MMH), especially lifting, leads to an increased risk of low back pain (LBP) [1-3]. In many occupations, it is difficult to avoid lifting of loads. For instance, European workers remain as exposed to $\mathrm{MMH}$ as they did 20 years ago: $33 \%$ of workers carry heavy loads at least a quarter of their working time [4]. Therefore, it is not surprising that emphasis has been given to optimise lifting techniques to prevent LBP $[5,6]$. Several lifting techniques have been advocated to reduce the load on the back. Probably the best known is the so-called 'leg lift' (lifting using a squat position). It has been questioned whether there are valid arguments for this technique from a biomechanical point of view [7]. In addition to advice on lifting techniques, assistive devices are often advocated to support the worker while lifting. This paper presents the results of the updated Cochrane review focusing on the effectiveness of MMH training and provision of assistive devices in preventing and treating LBP in workers [8]. Studies published since 2005 were included $[9,10]$.

\section{Methods}

An update of a Cochrane literature review [10] was performed using MEDLINE, EMBASE, CENTRAL, CINAHL, Nioshtic, CISdoc, Science Citation Index, and PsychLIT to November 2010. RCTs and CCTs were included.

\subsection{Participants and jobs}

Participants were working age adults (16 to 70 years, male or female) who were engaged in jobs

\footnotetext{
${ }^{*}$ Corresponding author. E-mail: p.p.kuijer@amc.nl, Tel no.: +31-20-566-5339
} 
with MMH to the extent that their risk for LBP was increased. The studies were supposed to include a description of the exposure. We had originally intended to only include studies with workers without LBP for the prevention studies. However, this proved to be too impractical because in many studies, some workers did have LBP, and it was too arbitrary a decision to define the maximum percentage of them that could be included for a study to still qualify as one of prevention. Therefore, we changed this inclusion criterion to workers who were not actively seeking treatment for current LBP. There were no restrictions on previous episodes of LBP.

\subsection{Types of interventions}

All interventions aimed at changing human behaviour in MMH or using MMH assistive devices were included. The interventions were divided into two groups according to the target of the intervention:

a) $\mathrm{MMH}$ techniques to decrease the mechanical load of MMH on the back,

b) the use of assistive devices to decrease the mechanical load of MMH on the back.

All interventions were included, regardless of the content, as long as the focus was on MMH techniques and the use of assistive devices, such as educational classes, individual training and instructions, posters, leaflets, videos, audiotapes, on the spot or on the job training, and combinations of several interventions. Any MMH assistive device could qualify as such, for example, hoists and other lifting aids, or sliding boards and other patient transfer aids.

Studies on interventions aimed at workplace adaptations were excluded because they go beyond advising workers about MMH techniques.

A broad range of $\mathrm{MMH}$ exposures were included (e.g. lifting, lowering, pulling and pushing), even though the amount of evidence on the relationship with back pain varies for the different exposures, e.g. the relationship with pushing and pulling, is not very clear. We dealt with this problem by classifying the interventions by the type of MMH exposure.

\section{Results}

Nine RCTs (20.101 employees) and nine CCTs (1280 employees) on prevention of LBP were included in this updated review: six more than in the previous version. All included studies focus on prevention of LBP; none on treatment. The studies were carried out in the time period between 1981 and 2010 with 11 of the 18 studies published in the past ten years. The studies were conducted in various geographical regions, with six studies from the US, three from Denmark, two from the Netherlands, and one each from Canada, China, France, Germany, Australia, Japan and Sweden.

\subsection{Participants and jobs}

Studies were conducted among the following occupations with exposure to back load: participants who lifted and moved patients (four RCTs and eight cohort studies), baggage handlers (two RCTs), construction workers (one RCT and one cohort study), postal workers who handled mail (one RCT) and workers in a distribution centre (one RCT). The number of participants varied from 131 to 12,772 in RCTs, and from 41 to 345 in cohort studies.

\subsection{Types of interventions}

The interventions varied from one session of simple advice on lifting to training once a week for two years. In some of the studies, traditional training was supported by follow-up and feedback at the workplace. The advocated lifting technique was usually not described in detail. One study measured the load on the back in real time and fed this back to the participant via a high pitched tone if the back was assessed to be overloaded. Another study gave nurses specific instructions about patient transfer techniques. Involvement of the supervisors in the intervention was clearly articulated in only a few studies, as was the encouragement to use available lifting aids. If reported, the number of participants in the training group was fewer than 15 employees. In four studies, the instructor was a trained colleague, in the other studies, the instructor was usually a professional in ergonomics. All interventions used an educational model that assumed that the information provided in the intervention would lead to a change in knowledge, attitude or skills. In addition to information, all interventions included the opportunity to practice the skills to some extent in either the educational setting or at follow-up in practice. None of the studies used a more elaborate model of change of health behaviour, such as the stages-of-change mod$\mathrm{el}$, the protection-motivation theory or the theory of reasoned action and planned behavior.

Studies compared training to no intervention $(n=4)$, professional education $(n=2)$, a video $(n=3)$, 
back belt use $(n=3)$ or exercise $(n=2)$. Other studies compared training plus lifting aids to no intervention $(n=3)$ and to training only $(n=1)$. The intensity of training ranged from a single educational session to very extensive personal biofeedback. Six RCTs and none of the CCTs had a high risk of bias.

\subsection{Effect on $L B P$}

There was moderate quality evidence in seven RCTs that training resulted in similar LBP as no intervention with an odds ratio of 1.17 (95\% Confidence Interval 0.68 to 2.02 ) and as minor advice with an odds ratio of 0.93 (95\% Confidence Interval 0.69 to 1.25). Also none of the other comparisons showed evidence of a preventive effect of training and provision of assistive devices on LBP.

\section{Discussion}

This review shows that training workers in proper $\mathrm{MMH}$ techniques and providing them with assistive devices are not effective interventions by themselves in preventing LBP. The strength of our review is that we only included studies with designs that are least susceptible to bias. In addition, we were able to compare the results to studies with less valid study designs. There were no differences in conclusions between the analyses from the RCTs and the cohort studies.

A possible explanation for not finding a result may be that the exposure to $\mathrm{MMH}$ is not high enough in the included jobs to be at risk for work-related LBP. In four of the included RCTs, the MMH exposure was related to lifting patients, four RCTs studied handling of mail or luggage and one RCT evaluated construction workers. Even though the nature of MMH exposure varied, the results of the studies were not different with respect to biomechanical exposures. All jobs studied had more than sufficient exposure to biomechanical strain to the back to leave ample room for alleviation by effective interventions.

Another possible explanation for not finding an effect is that the risk of LBP might be related, not to incorrect $\mathrm{MMH}$ techniques, but to other work-related factors inherent in the studied populations (e.g. nonneutral, bent or rotated trunk postures without manual handling, or psychosocial strain).
In order to be able to devise new and innovative ways to prevent work-related LBP, researchers need a better understanding of the causal chain between exposure to $\mathrm{MMH}$ at work and the development of LBP.

\section{Acknowledgements}

The source for external support is the COMMONWEALTH OF AUSTRALIA as represented by and acting through the Department of Employment and Workplace Relations. The Office of the Australian Safety and Compensation Council (OASCC) is the direct supporter. The Office of the ASCC resides within the Department and supports the Australian Safety and Compensation Council.

This review was made under the auspices of the Cochrane Back Review Group. We thank Rachel Couban for her assistance with the searches and Victoria Pennick for the editorial services.

\section{References}

[1] Kuiper J.I., Burdorf A., Frings-Dresen M.H., et al. Assessing the work-relatedness of nonspecific low-back pain. Scand J Work Environ Health. (2005), 31(3), 237-43.

[2] Takala, E.P. Lack of "statistically significant" association does not exclude causality. (2010). Spine J., 10(10), 944.

[3] Kuijer P.P., Frings-Dresen, M.H., Gouttebarge, V., et al. Low back pain: we cannot afford ignoring work. (2011). Spine J., 11(2), 164.

[4] Eurofound. Changes over time - First findings from the fifth European Working Conditions Survey. (2010) April 6 ${ }^{\text {th }}, 2011$ www.eurofound.europa.eu/surveys/ewcs/index.htm

[5] Straker, L.M. A review of research on techniques for lifting low-lying objects: 1. Criteria for evaluation. Work, (2002a), 19(1), 9-18.

[6] Straker, L.M. A review of research on techniques for lifting low-lying objects: 2. Evidence for a correct technique. Work, (2002b), 20(2), 83-96.

[7] Van Dieën, J.H., Hoozemans M.J., Toussaint, H.M. Stoop or squat: a review of biomechanical studies on lifting technique. (1999), Clin. Biomech., 14(10), 685-96.

[8] Verbeek J., Martimo K.P., Karppinen J., et al. Manual material handling advice and assistive devices for preventing and treating back pain in workers. (2011) Cochrane Database Syst Rev. 15(6):CD005958

[9] Martimo, K.P., Verbeek, J., Karppinen, J., et al. Effect of training and lifting equipment for preventing back pain in lifting and handling: systematic review. (2008), BMJ., 23, 429-31.

[10] Martimo, K.P., Verbeek, J., Karppinen, J.,et al. Manual material handling advice and assistive devices for preventing and treating back pain in workers. (2007), Cochrane Database Syst. Rev., 18(3), CD005958. 